\title{
Length of Endoprosthetic Reconstruction in Revision Knee Arthroplasty Is Associated With Complications and Reoperations
}

\author{
Jeffrey J. Barry MD, Zachary Thielen MD, David C. Sing BS, \\ Paul H. Yi MD, Erik N. Hansen MD, Michael Ries MD
}

Published online: 19 April 2016

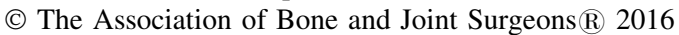

\begin{abstract}
Background Complex revision total knee arthroplasty (TKA) often calls for endoprosthetic reconstruction to address bone loss, poor bone quality, and soft tissue insufficiency. Larger amounts of segmental bone loss in the setting of joint replacement may be associated with greater areas of devascularized tissue, which could increase the risk of complications and worsen functional results.

Questions/purposes Are longer endoprosthetic reconstructions associated with (1) higher risk of deep infection; (2) increased risk of reoperation and decreased implant survivorship; or (3) poorer ambulatory status?

Methods This is a single-institution retrospective case series of nononcologic femoral endoprosthetic reconstructions for revision TKA from 1995 to $2013(n=32)$. Cases were categorized as distal $(n=17)$ or diaphyseal $(n=15)$ femoral reconstructions based on extension to or above the supracondylar metaphyseal-diaphyseal junction, respectively. Five patients from each group were lost to followup
\end{abstract}

One of the authors (MR), or a member of his or her immediate family, has or may receive payments or benefits, during the study period, an amount of more than USD 1,000,001, from Smith \& Nephew, Inc (Memphis, TN, USA).

All ICMJE Conflict of Interest Forms for authors and Clinical Orthopaedics and Related Research $\mathbb{R}$ editors and board members are on file with the publication and can be viewed on request.

Each author certifies that his or her institution approved the human protocol for this investigation, that all investigations were conducted in conformity with ethical principles of research, and that informed consent for participation in the study was obtained.

J. J. Barry, Z. Thielen, D. C. Sing, P. H. Yi, E. N. Hansen, M. Ries ( $\square)$

University of California, San Francisco, 500 Parnassus Avenue, MU320W, San Francisco, CA 94143, USA

e-mail: Michael.Ries@ucsf.edu; riesm@ orthosurg.ucsf.edu before 2 years (distal mean 4 years [range, 2-8 years]; diaphyseal mean $=6$ years [range, $2-16$ years]), and one of the 12 distal reconstructions and two of the 10 diaphyseal reconstructions had not been evaluated within the past 5 years. Clinical outcomes and ambulatory status (able to walk or not) were assessed through chart review by authors not involved in any cases. Prior incidence of periprosthetic joint infection was high in both groups (distal $=$ seven of 12 versus diaphyseal $=$ four of $10 ; p=0.670$ ).

Results Patients with diaphyseal femoral replacements were more likely to develop postoperative deep infections than patients with distal femoral replacements (distal $=$ three of 12 versus diaphyseal $=$ nine of $10 ; \mathrm{p}=0.004$ ) Implant survivorship (revision-free) for diaphyseal reconstructions was worse at 2 years (distal $=100 \%, 95 \%$ confidence interval $[\mathrm{CI}], 100 \%-100 \%$ versus diaphyseal $=$ $40 \%, 95 \% \mathrm{CI}, 19 \%-86 \% ; \mathrm{p}=0.001)$ and 5 years $($ distal $=$ $90 \%, 95 \%$ CI, $75 \%-100 \%$ versus diaphyseal $=30 \%, 95 \%$ $\mathrm{CI}, 12 \%-73 \% ; \mathrm{p}=0.001)$. Infection-free, revision-free survival (retention AND no infection) was worse for diaphyseal femoral replacing reconstructions than for distal femoral replacements at 2 years $($ distal $=70 \%, 95 \% \mathrm{CI}$, $48 \%-100 \%$ versus diaphyseal $=20 \%, 95 \%$ CI, 6\%-69\%; $\mathrm{p}=0.037)$ and 5 years $($ distal $=70 \%, 95 \% \mathrm{CI}, 48 \%-100 \%$ versus diaphyseal $=10 \%, 95 \% \mathrm{CI}, 2 \%-64 \% ; \mathrm{p}=0.012$ ). There was no difference with the small numbers available in proportion of patients able to walk (distal reconstruction $=$ eight of 11 versus diaphyseal $=$ seven of $10 ; p=1.000$ ), although all but one patient in each group required walking aids.

Conclusions Endoprosthetic femoral reconstruction is a viable salvage alternative to amputation for treatment of failed TKA with segmental distal femoral bone loss. In our small series even with substantial loss to followup and likely best-case estimates of success, extension proximal to 
the supracondylar metaphyseal-diaphyseal junction results in higher infection and revision risk. In infection, limb salvage remains possible with chronic antibiotic suppression, which we now use routinely for all femoral replacement extending into the diaphysis.

Level of Evidence Level III, therapeutic study.

\section{Introduction}

The number of primary and revision TKAs performed annually continues to increase with estimates of upward of 1.3 million primary TKAs and 125,000 revision TKAs to be performed per year by 2020 [13]. As indications for joint arthroplasty have expanded, there has been a disproportionate increase in the number of TKAs performed in younger patients [14]. These trends are expected to contribute to the rising number of revision arthroplasties performed each year. Berend et al. [3] described the concept of trading arthritic disease for "prosthetic disease," which represents all possible failure modes of a prosthetic implant during the life of the patient. Multiply revised TKAs for infection, fracture, or instability are not uncommon and represent a complex reconstructive challenge. With revision after revision, these patients can develop accompanying bone loss, poor bone quality, soft tissue insufficiency, instability, and infection, all of which further complicate efforts to achieve a successful outcome.

Endoprostheses (also known as megaprostheses or tumor prostheses) have been used with success in the orthopaedic oncology setting for decades in the setting of limb salvage after large bony resections. Their use in the nontumor setting is less common, accounting for only approximately $7 \%$ of all endoprosthetic reconstructions [16]. An endoprosthesis is an effective alternative to structural allograft reconstruction for revision TKA in the setting of severe femoral bone loss. The relative ease of insertion and early mobility it affords are attractive benefits, especially for older and frailer individuals $[5,9,10]$.

Numerous case series have been published for the use of endoprostheses in the revision arthroplasty setting [2, 7, 10-12, 15, 17, 19-21]. These series describe the outcomes related to individual types of reconstructions, ie, distal femoral or diaphyseal femoral. However, no study to date to the authors' knowledge has directly compared outcomes between these two types of reconstruction of different endoprosthetic length and bone replacement. It is unknown whether the amount of bone loss and size of the endoprosthesis are correlated to complication risk, infection, reoperation, and ambulatory status.

We therefore asked: (1) Are longer endoprosthetic reconstructions associated with higher risk of infection? (2)
Does increasing endoprosthetic length increase the risk of reoperation and decrease implant survivorship? (3) Is increasing length of an endoprosthesis associated with poorer ambulatory status?

\section{Patients and Methods}

An institutional review board-approved retrospective case series was performed consisting of all nononcologic femoral endoprosthetic reconstructions for revision TKA with distal femoral bone loss performed at a single tertiary care institution from 1995 to 2013. Cases were categorized as distal femoral replacement or diaphyseal femoral replacement. Patient demographics and outcome variables including number of prior surgeries, reoperations, infections, implant survival, and ambulatory status were collected by electronic medical record review.

Patients were initially screened for inclusion by keyword search of all operative notes of revision total joint arthroplasty cases (keywords: hinge, endoprosthesis, diaphyseal, diaphysis, distal femoral replacement, distal femoral arthroplasty, megaprosthesis, tumor prosthesis). A retrospective chart and radiograph review were then performed to identify all patients who had undergone endoprosthetic reconstruction for revision of a failed TKA. Cases were separated in groups by length of endoprosthetic reconstruction as follows: (1) distal femoral reconstructions, which included extension from the knee to the supracondylar metaphyseal-diaphyseal junction; or (2) diaphyseal femoral reconstructions, which included the supracondylar metaphyseal-diaphyseal junction to the proximal femoral diaphysis (Fig. 1). It is important to note that the reconstruction had to include the entirety of the metaphysis at a minimum to be a distal femoral reconstruction and then any extension further up the diaphysis constituted a diaphyseal reconstruction. Hinged revisions using resurfacing components with distal augments and/or metaphyseal cones did not therefore meet inclusion criteria.

Initial keyword search resulted in 573 potential cases. After chart and radiograph review, 32 cases in 32 patients of endoprosthetic reconstruction for nononcologic revision TKA were identified (17 distal femoral reconstruction, 15 diaphyseal femoral reconstruction). Five patients from each group did not satisfy the minimum length of followup of 2 years leaving a total of 22 reconstructions for analysis (12 distal, 10 diaphyseal). Of the 10 patients without long-term followup, one patient died in the immediate perioperative period (diaphyseal), two had died before 2-year followup (both diaphyseal), and seven patients (five distal, two diaphyseal) were lost before 2 years. Attempts to connect with the seven lost patients by available contact information was 


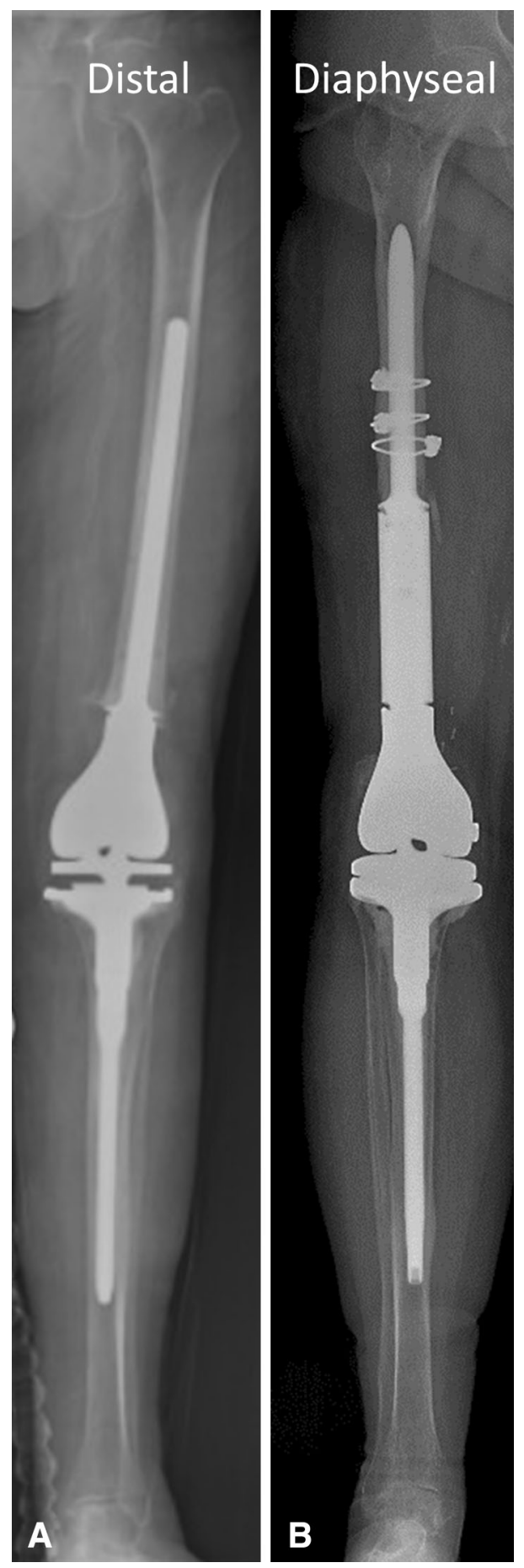

Fig. 1A-B Examples of the two lengths of endoprosthetic reconstruction studied for revision TKA with distal femoral bone loss are shown. A distal reconstruction extends from the knee to the supracondylar metaphyseal-diaphyseal junction (A) versus a diaphyseal reconstruction, which included cases from the supracondylar metaphyseal-diaphyseal junction to the proximal femoral diaphysis (B).

unsuccessful. Of the patients included for analysis, the mean length of followup was 4 years for distal reconstructions (range, 2-8 years) and 6 years for diaphyseal reconstructions (range, 2-16 years). Ten of the 12 distal and six of the 10 diaphyseal reconstructions were implanted after January 2006 and 11 of the 12 distal and eight of the 10 diaphyseal reconstructions had been evaluated within the last 5 years.

With the numbers available, patients in each group were not different in terms of age, sex, Charlson Comorbidity Index, mean number of prior procedures, history of soft tissue rotational flaps, history of periprosthetic joint infection (PJI), primary operative diagnosis at the time of revision reconstruction, or tibial component type (Table 1). A large proportion of patients across all groups had a history of PJI treated with a two-stage exchange at some point before (not necessarily immediately prior) the implantation of the endoprosthesis $($ distal $=$ seven of 12 versus diaphyseal $=$ four of $10 ; p=0.670$ ). The organism history for distal reconstruction were methicillin-resistant Staphylococcus aureus $\times 2$, methicillin-sensitive $S$ aureus, Enterococcus faecalis $\times 2$, Propionibacterium acnes, and Streptococcus. For the diaphyseal reconstruction group, prior organisms were methicillin-resistant $S$ aureus, Staphylococcus lugdunensis, Gram-positive rods, and Candida albicans. Additionally, no difference was seen in the proportion of endoprostheses implanted for segmental bone loss immediately after a two-stage exchange for active PJI $($ distal $=$ four of 12 versus diaphyseal $=$ three of $10 ; p=$ $0.867)$.

Total femoral replacements were not included in this study because the majority were related to failed THA and felt to represent a different clinical entity. Patients were excluded for any oncologic diagnoses given the inherent differences between patients undergoing revision arthroplasty and the oncologic population.

All patients selected had undergone reconstruction with an endoprosthesis for revision TKA in the nononcologic setting. Two fellowship-trained, Knee Society member, joint arthroplasty surgeons performed all cases included in analysis except for three (one distal and two diaphyseal reconstructions). For reference, from 2006 through 2013, the same two surgeons performed 1013 revision total knee procedures (average $>60$ cases each per year). It is our institution's practice to preserve as much viable bone as possible in all cases and use distal or diaphyseal segmental replacement only as a final reconstructive alternative to amputation. Despite practicing at a tertiary care center with frequent cases involving large amounts of bone loss, the number of endoprosthetic reconstructions performed accounted for only $2.4 \%$ of all revision total knees (24 cases including those lost to followup over the same time period of 2006-2013).

All infections were treated with a standard two-stage exchange protocol. At the initial explantation, all devitalized and compromised soft tissues and bone were 
Table 1. Patient characteristics

\begin{tabular}{llll}
\hline Characteristic & Distal femoral replacement & Diaphyseal femoral replacement \\
\hline Number & 12 & 10 \\
Age (years), mean (SD) & $63(12)$ & $64(10)$ & 0.802 \\
Sex, male:female & $4: 8$ & $3: 7$ & 1 \\
Charlson Comorbidity Index, mean (SD) & $3(1)$ & $4(4)$ & 0.574 \\
Number of prior procedures, mean (SD) & $4(4)$ & 4 \\
History of infection & 7 & 0 & 0.561 \\
Rotational flap & 2 & 9 \\
Tibial component & & 0 \\
Stemmed & 8 & 1 \\
Stemmed + augments & 2 & 3 \\
Proximal tibial replacement & 2 & 5 \\
Primary operative diagnosis & & 2 \\
Infection & 4 & 0.481 \\
Fracture & 4 & 4 & 0.328 \\
Aseptic loosening/instability & 4 & \\
\hline
\end{tabular}

thoroughly débrided followed by placement of a static antibiotic-impregnated cement spacer. A minimum of 6 weeks of targeted intravenous antibiotic was administered followed by a minimum 2 -week antibiotic holiday. Then serum erythrocyte sedimentation rate and C-reactive protein were drawn as well as aspiration and culture of joint fluid. Reimplantation was performed only with normalization of the serum inflammatory markers and sterile aspiration. Patients were given 24 hours of perioperative antibiotic prophylaxis with cefazolin, vancomycin, or cefazolin and vancomycin. Chronic long-term oral antibiotic therapy was not used routinely after revision with either distal or diaphyseal reconstruction. Postoperative infection was defined by the Musculoskeletal Infection Society criteria for PJI. A subset of patients who developed postoperative infections after their distal or diaphyseal replacement were treated with débridement, polyethylene liner exchange, and retention of components. These patients received intravenous antibiotics for 6 weeks followed by use of oral antibiotic therapy under the direction of an infectious disease consultant.

Once cases had been identified, retrospective chart and radiograph review was performed by study authors other than operating surgeons to identify variables of interest including age, sex, length of followup, number of procedures before endoprosthetic implantation, history of knee PJI before endoprosthetic reconstruction, surgical diagnosis at the time of endoprosthetic reconstruction, reoperation risk, endoprosthetic PJI risk, prevalence of chronic suppressive antibiotic use postoperatively, implant survival (defined as time to explant OR revision of endoprosthetic components), infection-free, revision-free implant survival (defined as time to diagnosis and débridement of infection OR explant OR revision of endoprosthetic components), and ambulatory status at most recent followup. Ambulation status was separated into able to walk (regardless of aid required) or full-time wheelchair user. Those who were able to walk were further divided into those without any aids and those that required aids (cane, crutches, or walker). Patient administrative records were reviewed to calculate Charlson Comorbidity Index without age adjustment using 16 comorbidities identified through International Classification of Diseases, $9^{\text {th }}$ Revision, Clinical Modification coding [18].

\section{Statistical Analysis}

Categorical variables were compared using Fisher's exact test or chi square when applicable. Continuous variables were compared using t-test or analysis of variance F-test when comparing more than three groups. Statistical significance was defined as $\mathrm{p}<0.05$. Kaplan-Meier survival curves were calculated with $95 \%$ CI included. Log-rank testing was used to determine significant differences in survival between groups. All statistical analysis was performed using R Version 3.0.2 (R-Project, R Foundation for Statistical Computing, Vienna, Austria).

\section{Results}

Patients with diaphyseal femoral replacements were more likely to develop deep infections after their reconstruction 
Table 2. Results

\begin{tabular}{llll}
\hline Outcome & Distal femoral replacement & Diaphyseal femoral replacement & $\mathrm{p}$ value \\
\hline Number & 12 & 10 & 0.004 \\
Endoprosthetic infection & 3 & 9 & 0.015 \\
New infection with no history of infection & $0 / 5$ & $4 / 6$ & 0.194 \\
Recurrent infection & $3 / 7$ & $3 / 4$ & 1 \\
Different organism & $1 / 1^{*}$ & 6 & 0.074 \\
Long-term antibiotics & 2 & 9 & 0.012 \\
All-cause reoperation rate & 4 & $4(3)$ & 0.389 \\
Subsequent number procedures, mean (SD) & $1(1)$ & 4 & 0.029 \\
Amputation & 0 & $7 / 10$ & 1 \\
Ambulatory & $8 / 11^{\dagger}$ & 1 & 1 \\
Ambulatory without assistive devices & 1 & & \\
\hline
\end{tabular}

* Two recurrent infections were culture-negative; ${ }^{\dagger}$ one patient had an unclear ambulatory status.

than were patients treated with distal femoral replacements $($ distal $=$ three of 12 versus diaphyseal $=$ nine of $10 ; p=$ 0.004; Table 2). Infection recurred after endoprosthetic implantation in patients with a history of PJI in three of seven distal and four of four diaphyseal reconstructions $(p=0.190)$. New infections occurred in previously aseptic limbs in zero of five distal and five of six diaphyseal reconstructions $(p=0.015)$. With the numbers available, the incidence of implant retention with débridement and long-term suppressive antibiotic therapy for limb salvage after postoperative infection were not different between the distal and diaphyseal reconstruction groups (distal $=$ two of 12 versus diaphyseal $=$ six of $10 ; p=0.074)$. Nearly all organisms responsible for postoperative infection in the diaphyseal group were coagulase-negative Staphylococcus species (seven of nine infections; other organisms were Streptococcus and Escherichia coli) compared with the distal femoral reconstruction group in which there was one E. faecalis infection and two culture-negative infections that met other Musculoskeletal Infection Society PJI criteria.

Implant survival (Kaplan-Meier survivorship free from explant or revision of components) of diaphyseal replacing reconstructions was worse than distal femoral replacing reconstructions at 2 years $($ distal $=100 \%, 95 \% \mathrm{CI}, 100 \%-$ $100 \%$ versus diaphyseal $=40 \%, 95 \% \mathrm{CI}, 19 \%-86 \% ; \mathrm{p}=$ $0.001)$ and 5 years $($ distal $=90 \%, 95 \%$ CI, $75 \%-100 \%$ versus diaphyseal $=30 \%, 95 \% \mathrm{CI}, 12 \%-73 \% ; \mathrm{p}=0.001$ ) (Fig. 2). Infection-free, revision-free survival (no infection, component revision or explant) was also worse for diaphyseal replacing reconstructions than for distal femoral replacements at 2 years (distal $=70 \%, 95 \% \mathrm{CI}, 48 \%-100 \%$ versus diaphyseal $=20 \%, 95 \% \mathrm{CI}, 6 \%-69 \% ; \mathrm{p}=0.037)$ and 5 years $($ distal $=70 \%, 95 \% \mathrm{CI}, 48 \%-100 \%$ versus diaphyseal $=10 \%, 95 \%$ CI, 2\%-64\%; $p=0.012$; Fig. 3).

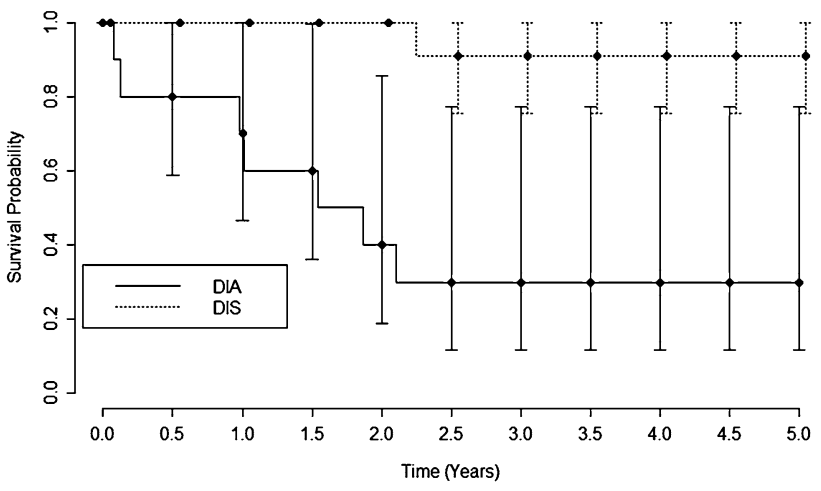

Fig. 2 Kaplan-Meier survival curve for implant survival (free from explant or component revision) comparing distal (DIS) and diaphyseal (DIA) femoral endoprosthetic reconstructions are shown. Bars represent the $95 \% \mathrm{CI}$.

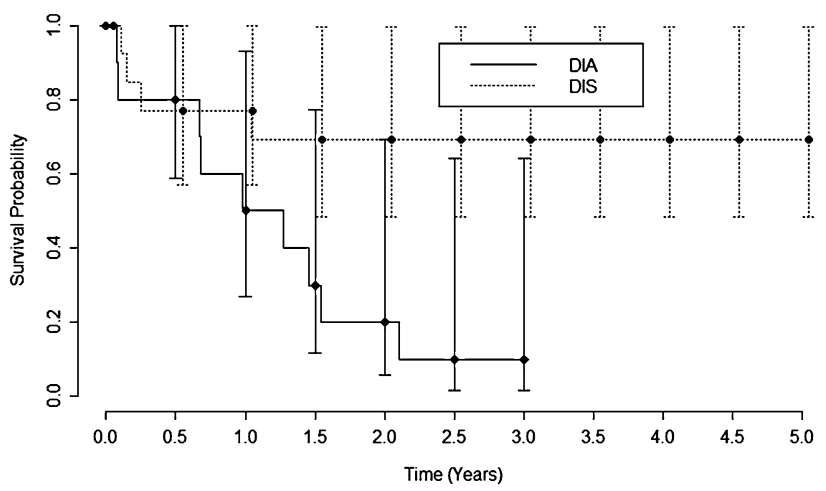

Fig. 3 Kaplan-Meier survival curve for infection-free, revision-free survival comparing distal (DIS) and diaphyseal (DIA) femoral endoprosthetic reconstructions are shown. Bars represent the 95\% CI.

The vast majority of reoperations were associated with infections $($ distal $=$ three of four versus diaphyseal $=$ eight of nine; $\mathrm{p}=1.000$ ). Only one patient from each group 
underwent a noninfectious reoperation (one distal reconstruction for patellar component revision, one diaphyseal reconstruction for a periprosthetic fracture).

Compared with distal femoral endoprostheses, with the numbers available, diaphyseal endoprostheses showed no difference in the likelihood a patient would regain the ability to walk (distal $=$ eight of 12 versus diaphyseal = seven of $10 ; p=1.000$ ) (Table 2). One patient from each group was able to ambulate without any assistive devices. The remainder of the ambulatory patients required a cane or walker for assistance. Above-knee amputations were ultimately performed on zero of 12 distal femoral replacements and four of 10 diaphyseal femoral replacements ( $\mathrm{p}=0.029)$. All patients who underwent amputation used wheelchairs to ambulate except one patient who used a walker after an above-knee amputation for infection.

\section{Discussion}

The use of endoprosthetics as a salvage option for complicated revision arthroplasty may become more commonplace as the number of revisions performed continues to increase. Prior series have typically analyzed a single implant type [2, 7, 10-12, 15, 17, 19-21]. To our knowledge, direct comparisons between different endoprosthesis subtypes for the treatment of distal femoral bone loss have not previously been performed. The purpose of this study was to evaluate if the length of the endoprosthesis implanted had an effect on outcomes with midterm followup. We found that prostheses that extended past the metaphyseal-diaphyseal junction of the distal femur were associated with higher risks of infection, reoperation, and implant revision.

The biggest limitation of endoprosthesis studies is the small number of patients given the rarity of surgical indications and use. Our institution's practice is to only use endoprosthetic reconstruction as a last resort for the most severe bone loss cases and so these cases represent only $2.4 \%$ of revision total knees. When combined with the retrospective nature of this work, detailed statistics such as multivariate analysis would be greatly underpowered. Ten patients (five from each group) were not available for 2-year followup with three of five of the diaphyseal patients confirmed dead before 2 years and the rest unable to be contacted. Additionally, of those with sufficient followup duration, one of 12 distal and two of 10 diaphyseal reconstructions had not been evaluated in the past 5 years. Given this substantial portion of the patients lost to followup, our already sobering results likely represent a bestcase scenario because those lost to followup are often faring worse. However, followup loss was equal between the two groups and a known problem in this older, frail population [2]. Other limitations include that our study spans nearly two decades with two main primary surgeons with potential confounding from implant design, surgical technique, and postoperative management. The three patients from other surgeons also increase this potential confounding. The wide study period may have affected the diaphyseal group more given four of 10 were performed before 2006 compared with only two of 12 of the distal reconstructions. Additionally, the study population is quite heterogeneous with many patients arriving at a salvage operation for a variety of reasons.

In our series, half of patients (11 of 22) had a history of PJI treated with a two-stage exchange at some stage before their endoprosthetic implantation. Seven of 22 had the twostage exchange immediately before endoprosthetic implantation. For comparison, previously reported infection history preimplantation has ranged from $16 \%$ to $20 \%$ for distal and $4 \%$ to $16 \%$ for diaphyseal endoprostheses $[10,11,20]$. Previously reported infection risk after endoprosthetic reconstruction of various lengths for revision arthroplasty in the nononcologic setting have varied from $13 \%$ to $35 \%$ for distal through total femoral replacements $[1,2,6,10-12,20]$. Our high proportion of patients with a PJI history compared with previously reported cohorts likely contributed to the high proportion of patients who developed postoperative infection overall in this series; however, we feel the bigger cases through larger exposures in patients with multiple comorbidities and compromised soft tissues likely all contribute to the higher prevalence in the diaphyseal group. Interestingly, nearly all reinfections occurred with different organisms and the majority of postoperative infections involved Staphylococcus epidermidis, a biofilm-producing organism. The standard practice for these replacements at our institution had previously been to use 24 hours of antibiotic postoperative prophylaxis. Interestingly, Berend et al. [3] reported on 59 total femoral replacements for nononcologic revision and had $14 \%$ infections at 5 years followup after using prolonged antibiotics in all patients despite a preoperative infection history in $24 \%$ of patients. Similar to their group, given the morbidity of infection in these implants, it is now our practice to place all patients with prostheses that extend into the diaphysis on empiric chronic antibiotic therapy postoperatively. Investigation of this prolonged antibiotic course as well as collaborative efforts with oncology colleagues on interventions like local antibiotic delivery or silver-coated prostheses warrants future attention [8, 22].

Compared with distal femoral replacement, diaphyseal femoral replacement was associated with decreased implant survival (explant or component revision) at 5 years postoperatively $($ distal $=90 \%, 95 \% \mathrm{CI}, 75 \%-100 \%$ versus diaphyseal $=30 \%, 95 \% \mathrm{CI}, 12 \%-73 \% ; \mathrm{p}=0.001)$. 
However, in our small series, this already sobering survival rate may still falsely estimate true "success," because there was a disparity in both groups between implant survival and infection-free, implant survival (cases without infection AND without revision/explant-Kaplan-Meier 5 years [distal $=70 \%, 95 \%$ CI, $48 \%-100 \%$ versus diaphyseal $=$ $10 \%, 95 \% \mathrm{CI}, 2 \%-64 \% ; \mathrm{p}=0.012)$. The difference comes from patients treated with multiple débridements and chronic antibiotic suppression given the morbidity associated with revision, staged exchange, or amputation. Although the implant "survives," it is difficult to argue these cases as success. Our revision-free survival at 5 years for distal reconstructions is in line with previously reported rates for endoprosthetics in the nontumor setting; however, revision risk for the diaphyseal reconstruction group was substantially higher. Reoperation or major complication prevalence has been reported as $13 \%$ to $59 \%$ for distal and $27 \%$ to $31 \%$ for diaphyseal reconstructions previously when reported separately $[2,3,7,10-12,15,19,20]$. However, as noted, our cohort's infection proportion before implantation was higher and all reoperations except for two were infection-related. Survivorship for noninfectious indications in revisions total joints have been noted to be better historically $[4,6]$.

Despite the high morbidity associated with endoprosthetic reconstruction for complex revision arthroplasty, the likelihood a patient would be able to walk at final followup was high if they had not undergone an amputation (14 of 17). Amputations only occurred in the diaphyseal group in our small series. When used as a salvage option, endoprosthetic reconstruction can offer an immediate mobility benefit over amputation, especially in the older, frail individuals in whom bone loss is often a problem and immobility can be fatal. Our ambulatory proportion at final followup is similar to those reported for other endoprosthetic reconstructions for revision arthroplasty such as Höll et al. [10] who reported 19 of 20 distal and diaphyseal endoprosthetic reconstructions were ambulatory, although 16 required a walker and 10 were homebound. Similarly, Amantullah et al. [1] reported 14 of 20 patients ambulatory at an average 73 months after total femoral replacement.

Endoprosthetic distal and diaphyseal femoral reconstruction can be used as a final salvage alternative to amputation for the treatment of failed TKA with segmental distal femoral bone loss. In our small limited series even with substantial loss to followup and likely best-case estimates of success, reconstructions with extension proximal to the supracondylar metaphyseal-diaphyseal junction result in higher infection and reoperation risk. This finding may be related to the greater area of bone and soft tissue devascularization and larger dead space associated with more proximal segmental replacement. Limb salvage in infected reconstructions remains possible, however, with infection source control and chronic antibiotic suppression. We now routinely use chronic empiric antibiotics for all endoprosthetic reconstructions extending into the diaphysis or higher in an effort to minimize the morbidity of infection in these salvage situations.

\section{References}

1. Amanatullah DF, Trousdale RT, Hanssen AD, Lewallen DG, Taunton MJ. Non-oncologic total femoral arthroplasty: retrospective review. J Arthroplasty. 2014;29:2013-2015.

2. Berend KR, Lombardi AV. Distal femoral replacement in nontumor cases with severe bone loss and instability. Clin Orthop Relat Res. 2009;467:485-492.

3. Berend KR, Lombardi AV, Mallory TH, Adams JB, Dodds KL. Total femoral arthroplasty for salvage of end-stage prosthetic disease. Clin Orthop Relat Res. 2004;427:162-170.

4. Clement ND, MacDonald D, Ahmed I, Patton JT, Howie CR. Total femoral replacement for salvage of periprosthetic fractures. Orthopedics. 2014;37:e789-795.

5. Davila J, Malkani A, Paiso JM. Supracondylar distal femoral nonunions treated with a megaprosthesis in elderly patients: a report of two cases. J Orthop Trauma. 2001;15:574-578.

6. Friesecke C, Plutat J, Block A. Revision arthroplasty with use of a total femur prosthesis. J Bone Joint Surg Am. 2005;87:26932701.

7. Haidukewych GJ, Springer BD, Jacofsky DJ, Berry DJ. Total knee arthroplasty for salvage of failed internal fixation or nonunion of the distal femur. J Arthroplasty. 2005;20:344-349.

8. Hardes J, von Eiff C, Streitbuerger A, Balke M, Budny T, Henrichs MP, Hauschild G, Ahrens H. Reduction of periprosthetic infection with silver-coated megaprostheses in patients with bone sarcoma. J Surg Oncol. 2010;101:389-395.

9. Harrison RJ, Thacker MM, Pitcher JD, Temple HT, Scully SP. Distal femur replacement is useful in complex total knee arthroplasty revisions. Clin Orthop Relat Res. 2006;446:113-120.

10. Höll S, Schlomberg A, Gosheger G, Dieckmann R, Streitbuerger A, Schulz D, Hardes J. Distal femur and proximal tibia replacement with megaprosthesis in revision knee arthroplasty: a limbsaving procedure. Knee Surg Sports Traumatol Arthrosc. 2012; 20:2513-2518.

11. Korim MT, Esler CN, Reddy VR, Ashford RU. A systematic review of endoprosthetic replacement for non-tumour indications around the knee joint. Knee. 2013;20:367-375.

12. Kostuj T, Streit R, Baums MH, Schaper K, Meurer A. Midterm outcome after mega-prosthesis implanted in patients with bony defects in cases of revision compared to patients with malignant tumors. J Arthroplasty. 2015;30:1592-1596.

13. Kurtz S, Ong K, Lau E, Mowat F, Halpern M. Projections of primary and revision hip and knee arthroplasty in the United States from 2005 to 2030. J Bone Joint Surg Am. 2007;89:780785 .

14. Losina E, Thornhill TS, Rome BN, Wright J, Katz JN. The dramatic increase in total knee replacement utilization rates in the United States cannot be fully explained by growth in population size and the obesity epidemic. J Bone Joint Surg Am. 2012;94: 201-207.

15. Mortazavi SM, Kurd MF, Bender B, Post Z, Parvizi J, Purtill JJ. Distal femoral arthroplasty for the treatment of periprosthetic fractures after total knee arthroplasty. J Arthroplasty. 2010; 25:775-780.

16. Pala E, Henderson ER, Calabrò T, Angelini A, Abati CN, Trovarelli G, Ruggieri P. Survival of current production tumor 
endoprostheses: complications, functional results, and a comparative statistical analysis. J Surg Oncol. 2013;108:403-408.

17. Peters CL, Hickman JM, Erickson J, Lombardi AV, Berend KR, Mallory TH. Intramedullary total femoral replacement for salvage of the compromised femur associated with hip and knee arthroplasty. J Arthroplasty. 2006;21:53-58.

18. Quan H, Sundararajan V, Halfon P, Fong A, Burnand B, Luthi JC, Saunders LD, Beck CA, Feasby TE, Ghali WA. Coding algorithms for defining comorbidities in ICD-9-CM and ICD-10 administrative data. Med Care. 2005;43:1130-1139.

19. Smith TH, Gad BV, Klika AK, Styron JF, Joyce TA, Barsoum WK. Comparison of mechanical and nonmechanical failure rates associated with rotating hinged total knee arthroplasty in nontumor patients. J Arthroplasty. 2013;28:62-67.e61.
20. Springer BD, Sim FH, Hanssen AD, Lewallen DG. The modular segmental kinematic rotating hinge for nonneoplastic limb salvage. Clin Orthop Relat Res. 2004;421:181-187.

21. Tillman R, Kalra S, Grimer R, Carter S, Abudu A. A custommade prosthesis attached to an existing femoral component for the treatment of peri- and sub-prosthetic fracture. $J$ Bone Joint Surg Br. 2006;88:1299-1302.

22. Wafa H, Grimer RJ, Reddy K, Jeys L, Abudu A, Carter SR, Tillman RM. Retrospective evaluation of the incidence of early periprosthetic infection with silver-treated endoprostheses in high-risk patients: case-control study. Bone Joint J. 2015;97:252257. 